\title{
Oral health knowledge and practice among administrative staff at Taibah university, Madina, KSA
}

\author{
Mohammad Sami Ahmad, Ahmed Bhayat, Khalid H. Al-Samadani ${ }^{1}$, Ziad Abuong ${ }^{2}$ \\ Departments of Preventive Dental Science, ${ }^{1}$ Restorative Dental Science, College of Dentistry, \\ Taibah University, ${ }^{2}$ Taibah University Clinics, Al Madinah Al Munawwarah, Kingdom of Saudi Arabia
}

\author{
Address for correspondence: \\ Dr. Mohammad Sami Ahmad, \\ Department of Preventive Dental \\ Science, College of Dentistry, Taibah \\ University, Al Madinah Al Munawwarah, \\ Kingdom of Saudi Arabia. \\ E-mail:msamiahmad@yahoo.com
}

\begin{abstract}
Background: Although the prevalence of dental caries is decreasing in developed countries, it is still increasing in developing countries. No studies have reported on the oral health status of adults in Saudi Arabia; the role models and parents for the younger generation. Materials and Methods: This was a cross-sectional study carried out between January and June 2012 and included 200 randomly chosen Taibah university staff members. Each participant received a self-administered questionnaire and consent form detailing the objectives and rational of the study. Results: The response rate was $74 \%$; mean age was 32.6 years and almost $90 \%$ had obtained higher educational qualifications. Nearly, half ( $48 \%$ ) cleaned their teeth in the morning and evening, $77 \%$ used a tooth stick and toothbrush and almost $90 \%$ used tooth paste regularly. Under two-thirds (61\%) visited the Dentist only when necessary and 13\% had never visited a Dentist. The treatment received included restorations (35\%), scaling and polishing (21\%) and extractions (18\%). Of those who did not visit the Dentist, $40 \%$ cited the high cost as the reason. The majority (78\%) were aware that sugar is harmful for the teeth. Under half (46\%) used tobacco and $36 \%$ had medical conditions. Those who visited the Dentist were more likely to brush twice daily $(P=0.04)$ and of those who brushed regularly, $50 \%$ knew the number of permanent teeth present $(P=0.04)$ and $57 \%$ were aware of the benefits of fluoride $(P=0.01)$. Conclusion: The majority of respondents had a poor level of knowledge regarding oral hygiene. This was reflected in their poor and inconsistent brushing habits and their lack of utilization of dental services.
\end{abstract}

Key words

Oral hygiene, restoration, Taibah university, tooth paste, tooth stick

\section{INTRODUCTION}

During the past 20 years, there has been a reduction in the prevalence of dental caries and periodontal diseases among the population of industrialized countries. ${ }^{[1]}$ This is primarily due to the changing pattern of living conditions, adoption of a healthy life-style, the proper use of fluoride and the improved oral health practices, which have been shown to be responsible for this downward trend. ${ }^{[1]}$ In contrast, dental caries and periodontal diseases are increasing in some developing countries especially, where preventive programs have not been implemented properly. ${ }^{[2]}$ In Saudi Arabia, little is known about the oral health and oral care behavior among the

\begin{tabular}{|l|l|}
\hline \multicolumn{2}{|c|}{ Access this article online } \\
\hline Quick Response Code: & Website: \\
\hline & www.ejgd.org \\
\cline { 2 - 3 } & \\
\hline
\end{tabular}

Saudi adult population. ${ }^{[3]}$ The current adult population is a role model for the younger generation and it is essential to identify patterns of behavior that may influence oral health outcomes for future generations. It is necessary to determine the current levels of knowledge attitudes and practices of these adults in order to introduce appropriate and practical oral health programs, which would target those behaviors responsible for high levels of disease. Analysis of oral health behavior of the population is essential for the specification of oral health messages as well as for the development of behavior modification strategies. The aim was to determine the oral health practice, knowledge and attitude among administrative staff members at Taibah university, Madinah.

\section{MATERIALS AND METHODS}

This was a cross-sectional descriptive study carried out over a period of 6 months from January until June 2012. A total of 200 staff members were randomly selected form the official university staff data base. All were males as this was a male university. This represented a sample of $10 \%$ of permanent staff members employed on a full 
time basis. Each participant received a self-administered questionnaire and consent form detailing the objectives and rationale of the study. The questionnaires were collected either the same day or on the next day and those who did not complete the forms or were not available were asked to complete it the following day. The questionnaire consisted of 13 questions and was in the Arabic language. It was developed from a thorough the literature review of similar articles and relevant questions were included. The questionnaire was piloted amongst five dental assistants to identify any possible problems in its completion. The questionnaire was divided into various sections including demographics, knowledge and practice of oral habits, frequency of dental visits and medical status. Data was entered and the analysis was carried out using the SPSS 15.

\section{RESULTS}

Of the total 200 questionnaires that were delivered, 148 were returned $(74 \%)$. The mean age was $32.6( \pm 9.1)$ years and $89(60 \%)$ completed secondary school and had obtained higher qualifications.

Table 1 shows the responses obtained related to oral hygiene practice. Almost half (48\%) reported to clean their teeth both in the morning and in the evening, $77 \%$ used both the tooth stick (Miswak) and toothbrush and almost $90 \%$ used tooth paste regularly. Less than half (44\%) of participants used toothpicks regularly while only $16 \%$ reported to be using the dental floss on a regular basis.

Table 2 shows the reasons for visiting the Dentist and the type of treatment received. Almost 61\% reported to visit the Dentist only when necessary and $13 \%$ had never visited a Dentist before. Of those who visited the Dentist, the most common type of treatment received at the last visit was restorations (35\%), scaling and polishing (21\%) and extractions (18\%). Of those who did not visit the Dentist in the past year, the most common reasons were the high-cost (40\%), fear (24\%) and a lack of time (23\%).

Table 3 reports the levels of knowledge amongst the respondents. When asked about their dental knowledge, $22 \%, 33 \%$ and $34 \%$ answered correctly regarding the number of deciduous and permanent teeth present and the age of eruption for the first permanent tooth respectively. The majority $(78 \%)$ were aware that sugar is harmful for the teeth and almost all respondents $(92 \%)$ who had knowledge of fluoride felt that toothpaste contained fluoride, which was beneficial in preventing dental caries.

Table 4 displays the smoking prevalence and medical conditions reported by the participants. Just under half (46\%) used tobacco regularly and the majority (79\%) used cigarettes. Almost 21\% used water pipes (shisha) and of those who smoked cigarettes, $57 \%$ smoked more

\begin{tabular}{lcc}
\hline Table 1: Oral health practices & $\mathbf{N}$ & $\%$ \\
\hline Frequency of cleaning teeth $(n=148)$ & & \\
Morning only & 23 & 15.5 \\
Evening only & 18 & 12.0 \\
Morn. and even both & 70 & 47.5 \\
Occasionally & 37 & 25.0 \\
Instruments used for cleaning teeth $(n=148)$ & & \\
Brush & 31 & 21.0 \\
Finger & 3 & 2.0 \\
Miswak+brush & 114 & 77.0 \\
Types of dentifrices used ( $n=148)$ & & \\
Tooth paste & 128 & 86.5 \\
Tooth powder & 2 & 1.5 \\
Nothing & 12 & 8.0 \\
Others & 6 & 4.0 \\
Frequency of other oral hygiene aids used for cleaning $(n=148)$ & \\
Toothpick & 65 & 44.0 \\
Dental floss & 24 & 16.0 \\
Inter dental brush & 24 & 16.0 \\
Others & 23 & 15.5 \\
Nothing & 12 & 8.5 \\
\hline
\end{tabular}

\begin{tabular}{lcc}
\hline \multicolumn{2}{l}{ Table 2: Reasons for visiting the dentist and types of } \\
treatment received & N & $\%$ \\
\hline Frequency of dental visits $(n=148)$ & 14 & \\
After every 6 months & 7 & 9.5 \\
After every year & 90 & 4.5 \\
When needed & 18 & 61.0 \\
Occasionally & 19 & 12.0 \\
Never & & 13.0 \\
Types of treatment provided ( $n=111)$ & 15 & \\
Medicine only & 38 & 13.5 \\
Dental filling & 23 & 34.5 \\
Scaling and polishing & 20 & 20.5 \\
Extraction & 15 & 18.0 \\
Combination of treatment & & 13.5 \\
Reasons for avoiding dental visits $(n=148)$ & & \\
Fear & 35 & 23.5 \\
High cost & 59 & 40.0 \\
Lack of time & 34 & 23.0 \\
Not replied & 20 & 13.5 \\
\hline
\end{tabular}

than 10/day. Almost 36\% of the respondents had some form of medical condition and the most common amongst them was hypertension (24\%), diabetes (16\%), cardiac conditions (5\%) and others (52\%).

There was no statistically significant relationship between the variables, but there were some correlations that were significant. Those who claimed to visit the Dentist regularly were more likely to brush their teeth twice daily compared with those who did not visit the Dentist $(P=0.04)$. Of those who brushed regularly, 


\begin{tabular}{|c|c|c|}
\hline \multicolumn{3}{|c|}{ Table 3: Oral health knowledge } \\
\hline & $N$ & $\%$ \\
\hline \multicolumn{3}{|c|}{ Knowledge regarding the number of deciduous teeth } \\
\hline Correct & 33 & 22.5 \\
\hline Incorrect & 115 & 77.5 \\
\hline \multicolumn{3}{|c|}{ Knowledge regarding the number of permanent teeth } \\
\hline Correct & 49 & 33.0 \\
\hline Incorrect & 99 & 67.0 \\
\hline \multicolumn{3}{|c|}{$\begin{array}{l}\text { Knowledge regarding the age at which the first permanent molar } \\
\text { erupts }\end{array}$} \\
\hline Correct & 51 & $34 \cdot 5$ \\
\hline Incorrect & 97 & $65 \cdot 5$ \\
\hline \multicolumn{3}{|c|}{ Opinion about the role of sugar in dental caries } \\
\hline Cause caries & 115 & 78.0 \\
\hline Not cause caries & 18 & 12.0 \\
\hline Don't know & 15 & 10.0 \\
\hline \multicolumn{3}{|c|}{ Knowledge regarding the effects of fluoride on dental caries } \\
\hline Correct & 93 & 63.0 \\
\hline Incorrect & 55 & 37.0 \\
\hline \multicolumn{3}{|c|}{ Knowledge regarding the sources of fluoride } \\
\hline Tooth paste & 86 & 92.5 \\
\hline Drinking water & 3 & 3.5 \\
\hline Sea food & 1 & 0.5 \\
\hline Salt & 3 & 3.5 \\
\hline
\end{tabular}

\begin{tabular}{lcc}
\hline $\begin{array}{l}\text { Table 4: Prevalence of tobacco use and medical } \\
\text { conditions }\end{array}$ & N \\
& & \\
\hline Use of tobacco $(n=148)$ & 67 & 45.3 \\
Yes & 81 & 54.7 \\
No & & \\
Types of tobacco $(n=67)$ & 53 & 79.0 \\
Cigarette & 14 & 21.0 \\
Water pipe (shisha) & \\
Frequency of cigarette consumption per day $(n=53)$ & 43.0 \\
Less than 10 & 23 & 57.0 \\
More than 11 & 30 & \\
Prevalence of medical conditions $(n=148)$ & & 16.5 \\
Diabetes & 9 & 24.0 \\
Hypertension & 13 & 5.5 \\
Heart problem & 3 & 2.0 \\
Renal problem & 1 & 52.0 \\
Other problem & 28 & 64 \\
No problem & 94 & \\
\hline
\end{tabular}

$50 \%$ knew the correct answer regarding the number of permanent teeth present $(P=0.04)$ of these $57 \%$ were aware of the benefits of fluoride $(P=0.01)$.

\section{DISCUSSION}

The sample comprised only of administrative staff members as all of these are Saudi nationals and the objective of the study was to determine the oral health knowledge and habits amongst the local community.
The high response rate was expected as the investigators continually went to the respondents and asked for the questionnaires. In many cases, the investigators waited for the questionnaire after handing it to the respondent.

Almost two-thirds (60\%) had completed a post graduate diploma or degree. The majority of the staff was involved with human resources, contract approvals and liaising with foreign countries for recruitment purposes. As a result skills and training in management, accounting, labor practices and passport control and immigration procedures were essential for their normal duties.

Only half reported to clean their teeth both in the morning and evening and 25\% reported to clean their teeth "occasionally." This is considerably lower compared with studies in other countries, which showed that between $75 \%$ and $89 \%$ of adult brush twice a day. ${ }^{[4,5]}$ In Saudi Arabia, the tooth stick is commonly used to clean teeth, in this sample the prevalence of the tooth stick was as high as $77 \%$ and the cleaning is carried out regularly throughout the day. It could be possible that the respondents felt that the tooth stick is adequate for cleaning teeth and hence the toothbrush is not required regularly. Although $90 \%$ did claim to use a toothbrush and paste, this response could be as a result of response acquiescence, the respondents were aware that the investigators were Dentists and hence choose the "correct" answer.

Almost half (44\%) of participants used toothpicks regularly, this was considerably higher compared to a Danish study, ${ }^{[6]}$ which reported that $28 \%$ used toothpicks regularly. This could be attributed to the diet of Saudis, which includes lots of meat and chicken compared with the Danish community. The sample in Saudi was also much younger than that of the Danish study, which could indicate the Saudi sample having more teeth per person compared to more edentulous patients in the other study.

Only $16 \%$ reported to be using the dental floss on a regular basis, this was similar to the Danish study (11\%) and an American study, ${ }^{[7]}$ which reported a prevalence of between $8 \%$ and $14 \%$ of regular usage.

Almost $61 \%$ reported to visit the Dentist only when necessary, which was lower than another similar study in Saudi, which showed that $89 \%$ attended the Dentist only for emergency care. ${ }^{[8]}$ The authors in the previous study concluded that the levels of education and knowledge regarding oral health were still relatively low and hence many people were fearful and hesitant to visit the Dentist unless they were in severe pain. This was confirmed by the type of treatment patients reported to have received at the last visit, mostly restorations (34\%) and scaling and polishing (21\%) and extractions (18\%). It seems that the population is similar as one of the most common reasons cited for not attending was fear. 
Other reasons were the high cost $(40 \%)$ and a lack of time $(23 \%)$. Although there are free dental services offered at the university, many of the staff reported that the high cost was one of the deterrents in visiting the Dentist. This could be due to the fact that many staff members cannot access the clinic, which is open from 9.00 am until $4.00 \mathrm{pm}$ as they are working during these hours. They would have to seek dental treatment after hours, which is generally in the private sector and, which is expensive.

When asked about their dental knowledge, regarding eruption of teeth and the number of teeth present, less than 33\% could answer all the questions correctly and this confirms the results of Al-Otaibi and Angmar-Månsson (2004) ${ }^{[8]}$ who concluded that the levels of knowledge related to oral health is poor amongst many Saudis. However, in relation to other knowledge based questions regarding the relationship of fluoride and sugars to dental caries, a high percentage of respondents chose the correct answer. This improvement could be attributed to an increase in awareness through mass media related to basic oral health information, which was not present 10 years ago when Al-Otaibi conducted his study. Saudi Arabia has been developing rapidly in the past few years and adults have been exposed to internet, television and radio which regularly highlight the importance of oral health and the factors responsible for its health and disease.

Just under half (46\%) used tobacco regularly; this was similar to the results of published by Bassiony $(2009)^{[9]}$ who reported smoking prevalence of between $12 \%$ and $52 \%$ amongst adults in Saudi Arabia. Almost 21\% used water pipes on a regular basis; this was slightly higher than the $10 \%$ reported by Al Moamary et al. $(2012)^{[10]}$ amongst Saudi adolescents. Water pipe smoking has become increasingly popular in Saudi Arabia both amongst males and females and it has become a major public health concern. Amin et al. (2010) ${ }^{[1]}$ reported that half of his adolescent sample from Al Hossa (Saudi Arabia) was of the opinion that water pipes are less harmful than cigarettes and this may be responsible for the increase in the water pipe utilization.

Almost a third of the respondents had some form of medical condition. The most common medical conditions were no surprise as studies have shown a high prevalence of type 2 diabetes in Saudi Arabia, ${ }^{[12]}$ which has been linked to obesity, hypertension and cardiac conditions. Some of the reasons responsible for the high prevalence of diabetes in Saudi Arabia include rapid urbanization and changes in diet, aging populations as a result of an increase in access and availability of health services and obesity due to a decrease in physical activity.

Those who claimed to visit the Dentist regularly were more likely to brush their teeth twice daily compared to those who did not visit the Dentist. This showed that Dentists are providing valuable oral hygiene information and motivation for patients to try and improve their oral health habits. Those who brushed regularly had more oral health knowledge compared to those who were not brushing regularly. This confirmed that those who went to the Dentist received valuable oral health knowledge and this helped in improving and maintaining oral hygiene practices.

\section{CONCLUSION}

The majority of respondents had a poor level of knowledge regarding oral hygiene. This was reflected in their poor and inconsistent brushing habits and their lack of utilization of dental services. The authors therefore recommend the initiation of an oral hygiene awareness program amongst staff members at the university to improve their oral health knowledge and practices. Further studies can then be carried out to determine the effect of this programs.

\section{REFERENCES}

1. Zhu L, Petersen PE, Wang HY, Bian JY, Zhang Bx. Oral health knowledge, attitudes and behaviour of adults in China. Int Dent $\mathrm{J}$ 2005;55:231-41.

2. World Health Organization: Global Oral Health Data Bank. Geneva: WHO; 2002.

3. Farsi JM, Farghaly MM, Farsi N. Oral health knowledge, attitude and behaviour among Saudi school students in Jeddah city. J Dent 2004;32:47-53.

4. Zeedyk MS, Longbottom C, Pitts NB. Tooth-brushing practices of parents and toddlers: A study of home-based videotaped sessions. Caries Res 2005;39:27-33.

5. Aunger R. Tooth brushing as routine behavior. Int Dent $\mathrm{J}$ 2007;57:364-76.

6. Christensen LB, Petersen PE, Krustrup U, Kjøller M. Self-reported oral hygiene practices among adults in Denmark. Community Dent Health 2003;20:229-35.

7. Segelnick SL. A survey of floss frequency, habit and technique in a hospital dental clinic and private periodontal practice. N Y State Dent J 2004;70:28-33.

8. Al-Otaibi M, Angmar-Månsson B. Oral hygiene habits and oral health awareness among urban Saudi Arabians. Oral Health Prev Dent 2004;2:389-96.

9. Bassiony MM. Smoking in Saudi Arabia. Saudi Med J 2009;30:876-81.

10. Al Moamary MS, Al Ghobain MA, Al Shehri SN, Alfayez AI, Gasmelseed AY, Al-Hajjaj MS. The prevalence and characteristics of water-pipe smoking among high school students in Saudi Arabia. J Infect Public Health 2012;5:159-68.

11. Amin TT, Amr MA, Zaza BO, Suleman W. Harm perception, attitudes and predictors of waterpipe (shisha) smoking among secondary school adolescents in Al-Hassa, Saudi Arabia. Asian Pac J Cancer Prev 2010;11:293-301.

12. Ginter E, Simko V. Type 2 diabetes mellitus, pandemic in $21^{\text {st }}$ century. Adv Exp Med Biol 2012;771:42-50.

How to cite this article: Ahmad MS, Bhayat $\mathrm{A}$, Al-Samadani $\mathrm{KH}, \mathrm{Abuong} \mathrm{Z}$. Oral health knowledge and practice among administrative staff at Taibah university, Madina, KSA. Eur J Gen Dent 2013;2:308-11.

Source of Support: Nil, Conflict of Interest: None declared. 